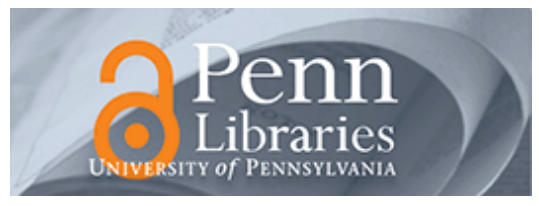

University of Pennsylvania

ScholarlyCommons

Operations, Information and Decisions Papers

Wharton Faculty Research

6-1992

\title{
A Priori Inequalities for the Euclidean Traveling Salesman
}

Timothy. L. Snyder

John M. Steele

University of Pennsylvania

Follow this and additional works at: https://repository.upenn.edu/oid_papers

Part of the Geometry and Topology Commons, and the Other Mathematics Commons

\section{Recommended Citation}

Snyder, T. L., \& Steele, J. M. (1992). A Priori Inequalities for the Euclidean Traveling Salesman. Proceeding SCG '92 Proceedings of the eighth annual symposium on Computational geometry, 344-349.

http://dx.doi.org/10.1145/142675.142745

This paper is posted at ScholarlyCommons. https://repository.upenn.edu/oid_papers/242

For more information, please contact repository@pobox.upenn.edu. 


\title{
A Priori Inequalities for the Euclidean Traveling Salesman
}

\author{
Abstract \\ It is proved that there are constants $c 1, c 2$, and $c 3$ such that for any set $S$ of $n$ points in the unit square \\ and for any minimum-lengths of T of $S(1)$ the sum of squares of the edge lengths of $T$ is bounded by $c 1$ \\ $\log n,(2)$ the sum of edge lengths of any subset $E$ of $T$ is bounded by $c 2|E| 1 / 2$, and (3) the number of \\ edges having length $t$ or greater in $T$ is at most $c 3 / t 2$. The second and third bounds are independent of \\ the number of points in $\mathrm{S}$, as well as their locations. Extensions to dimensions $\mathrm{d}>2$ are also sketched. The \\ presence of the logarithmic term in (1) is engaging because such a term is not needed in the case of the \\ minimum spanning tree and several analogous problems, and, furthermore, we know that there always \\ exists some tour of $S$ (which perhaps does not have minimal length) for which the sum of squared edges \\ is bounded independently of $n$.

\section{Disciplines} \\ Geometry and Topology | Other Mathematics
}




\title{
A Priori Inequalities for the Euclidean Traveling Salesman
}

\author{
Timothy Law Snyder ${ }^{1}$ \\ Department of Computer Science \\ Georgetown University \\ Washington, DC 20057 \\ J. Michael Steele ${ }^{2}$ \\ Department of Statistics \\ The Wharton School \\ University of Pennsylvania \\ Philadelphia, PA 19104
}

\begin{abstract}
It is proved that there are constants $c_{1}, c_{2}$, and $c_{3}$ such that for any set $S$ of $n$ points in the unit square and for any minimum-length tour $T$ of $S(1)$ the sum of squares of the edge lengths of $T$ is bounded by $c_{1} \log n$, (2) the sum of edge lengths of any subset $E$ of $T$ is bounded by $c_{2}|E|^{1 / 2}$, and (3) the number of edges having length $t$ or greater in $T$ is at most $c_{3} / t^{2}$. The second and third bounds are independent of the number of points in $S$, as well as their locations. Extensions to dimensions $d>2$ are also sketched.

The presence of the logarithmic term in (1) is engaging because such a term is not needed in the case of the minimum spanning tree and several analogous problems, and, furthermore, we know that there always exists some tour of $S$ (which perhaps does not have minimal length) for which the sum of squared edges is bounded independently of $n$.
\end{abstract}

1 Research supported in part by Georgetown University 1991 Summer Research Award.

2 Research supported in part by the following grants: NSF DMS88-12868, NSA MDA904-89-H-2034, AFOSR89-0301, and DAAL03-89-G-0092.

Permission to copy without fee all or part of this material is granted provided that the copies are not made or distributed for direct commercial advantage, the ACM copyright notice and the title of the publication and its date appear, and notice is given that copying is by permission of the Association for Computing Machinery. To copy otherwise, or to republish, requires a fee and/or specific permission.

\section{Introduction}

The purpose of this paper is to provide a priori bounds on quantities related to the edge lengths of an optimal traveling salesman (minimum-length) tour through $n$ points in the unit square. By a priori, we mean that the bounds are independent of the locations of the points. Studies of a priori bounds were initiated by Verblunsky (1951) and Few (1955). Few showed that for any set $S$ of $n$ points in the unit square, the length of an optimal traveling salesman tour of $S$ is at most $\sqrt{2 n}+1.75$. Few's result led to a series of improvements, culminating in Karloff (1989), where it was shown that Few's constant could be reduced to less than $\sqrt{2}$. Our results continue in this tradition by giving a priori inequalities for three other quantities related to the edge lengths of an optimal traveling salesman tour.

The interest in and subtlety of our inequalities comes from the fact that, in contrast to the minimum spanning tree (MST) problem, optimal solutions to the traveling salesman problem (TSP) are not invariant under monotone transformations of the edge weights. Before giving further details on this connection and other related work, we state our main results. We let $|e|=|x-y|$ denote the Euclidean length of the edge 
$e=\{x, y\}$ with vertices $x$ and $y$ in $\mathbb{R}^{2}$, and, in settings where the order of the edges of an optimal tour is not important, we represent a traveling salesman tour by the edge set $\left\{e_{1}, e_{2}, \ldots, e_{n}\right\}$. In what follows, an "optimal" traveling salesman tour is a tour that is of minimum length when using Euclidean edge weights.

Our first theorem bounds the sum of squared edge lengths of any optimal traveling salesman tour.

Theorem 1. There exists a constant $0<c_{1}<\infty$ such that if $T=\left\{e_{1}, e_{2}, \ldots, e_{n}\right\}$ is an optimal traveling salesman tour of $\left\{x_{1}, x_{2}, \ldots, x_{n}\right\} \subset[0,1]^{2}$ and if $n \geq 2$, then

$$
\sum_{i=1}^{n}\left|e_{i}\right|^{2} \leq c_{1} \log n .
$$

Theorem 2 is a bound on the number of edges that are of length $t$ or greater.

Theorem 2. There exists a constant $0<c_{2}<\infty$ such that, if $\nu(n, t)$ is the number of $e_{i} \in T$ such that $\left|e_{i}\right| \geq t$, then for all $t>0$ and $n \geq 1$,

$$
\nu(n, t) \leq c_{2} / t^{2}
$$

Corollary 3 gives a bound on the total length of any $k$-edge subset of an optimal TSP tour.

Corollary 3. There exists a constant $0<c_{3}<\infty$ such that, if $E=\left\{e_{i_{1}}, e_{i_{2}}, \ldots, e_{i_{k}}\right\} \subseteq T$, then

$$
\sum_{i \in E}\left|e_{i}\right| \leq c_{3} \sqrt{k}
$$

It is interesting to compare these results to their minimum spanning tree analogues. Steele and Snyder (1989) proved MST analogues to (1.2) and (1.3), but these proofs were predicated on a solution to the MST problem via a greedy algorithm, hence were not applicable to the TSP. The best TSP analogue to (1.3) was thus $\nu_{\mathrm{TSP}}(n, t) \leq c_{\mathrm{TSP}} \sqrt{n} / t$, for some constant $c_{\mathrm{TSP}}$. The bounds (1.2) and (1.3), however, are independent of $n$, the number of points, as well as the locations of the points. For this reason, we say that the significantly improved inequalities (1.2) and (1.3) are fully a priori.
Inequalities like (1.1) are important in simulations and investigations in which the square root computations required for Euclidean lengths are deemed to be too expensive (c.f. the discussion in Steele (1990)). It was observed in Steele (1990) by an application of the spacefilling curve heuristic that one could obtain a result like (1.1) for the MST, but without the logarithmic factor. Though this result makes the logarithmic term of (1.1) seem disappointing, the present bound can still be of service in many applications where a fully a priori inequality would be used.

A further subtlety in (1.1) is that we can easily show there always exists some (not necessarily optimal) tour $T^{\prime}$ of $S=\left\{x_{1}, x_{2}, \ldots, x_{n}\right\} \subset[0,1]^{2}$ for which $\sum_{e \in T^{\prime}}|e|^{2} \leq c \log n$, where $c$ is a constant. This can be shown by observing via a pigeonhole argument that there exist $x_{i}$ and $x_{j} \in S$, where $i \neq j$, such that $\left|x_{i}-x_{j}\right|$ is at most a constant times $n^{-1 / 2}$, then proceeding by an obvious induction. In fact, it has been known for some time that one can do considerably better; there is a constant $c^{\prime}$ and a tour $T^{\prime}$ of $S$ such that, for all $n \geq 2, \sum_{e \in T^{\prime}}|e|^{2} \leq c^{\prime}$. This can be obtained via the spacefilling heuristic as noted in the discussion of the MST, or it can be obtained by appropriately generalizing the Pythagorean Theorem (Neumann (1982)).

The sticky issue for the TSP is that, though there is some tour $T^{\prime}$ that makes $\sum_{e \in T^{\prime}}|e|^{2}$ particularly small, there is no compelling reason to believe that a traveling salesman tour $T$ that minimizes $\sum_{e \in T}|e|$ will do nearly so well. Because of the matroidal properties of the MST, such issues do not arise in its analysis. Analyzing the optimal TSP is much more difficult.

At present, we do not know of a way to remove the logarithmic factor in (1.1), nor do we have a lower bound that proves the necessity of the logarithmic factor. In the final section, we will comment further on this as well as problems concerning points in $[0,1]^{d}$ for dimension $d>2$. In Section 2, we prove two technical results that are applied in Section 3 to prove our main results.

This extended abstract is an abridged version of 
the full paper. Some details have been removed and the original numberings of equations and figures have been retained.

\section{Edge Lemmas}

The second lemma of this section explicates a property of non-intersecting edges in an optimal TSP tour and will be useful in the next section, where we prove our main results. Our first lemma gives a simple geometrical bound concerning quadrilaterals that assists the proof of the second lemma. In the statement of Lemma 1, the term "diagonal" is used to denote a segment connecting non-adjacent vertices of a quadrilateral, regardless of whether the quadrilateral is convex.

Lemma 1. Let $L_{1}$ and $L_{2}$ be two non-intersecting line segments satisfying $r \leq\left|L_{i}\right| \leq \beta r$, where $\beta>1$. Suppose the midpoints of $L_{1}$ and $L_{2}$ are separated by distance $\lambda$. If the endpoints of $L_{1}$ and $L_{2}$ are joined to form a quadrilateral $Q$ with sides $L_{1}, L_{2}, S_{1}$, and $S_{2}$, then $\left|S_{i}\right| \leq \frac{1}{2}(\beta-1) r+3 \lambda$ for $i=1,2$. Moreover, the lengths of the diagonals of $Q$ are bounded by $\beta r+\lambda$.

Proof.

The proof of Lemma 1, which relies on relatively simple geometric observations, is omitted for the extended abstract. It can be provided on request.

Lemma 2. Let $\left\{e_{1}, e_{2}, \ldots, e_{n}\right\}$ denote the edges of an optimal traveling salesman tour of $\left\{x_{1}, x_{2}, \ldots, x_{n}\right\} \subset$ $\mathbb{R}^{2}$. For each $e_{i}$ satisfying $r \leq\left|e_{i}\right| \leq \beta r$, let $D_{i}$ denote the disk of radius $\alpha\left|e_{i}\right|$ centered at the midpoint of $e_{i}$, where $\alpha=1 / 22$ and $\beta=3 / 2$. If $D_{i_{1}}, D_{i_{2}}$, and $D_{i_{3}}$ are three disks such that no pair of the edges $e_{i_{1}}, e_{i_{2}}$, and $e_{i_{3}}$ has a vertex in common, then, for all $r>0$, the intersection $D_{i_{1}} \cap D_{i_{2}} \cap D_{i_{3}}$ is empty.

\section{Proof.}

Without loss, we let $i_{j}=j$ for $j=1,2,3$. We show that if $D_{1}, D_{2}$, and $D_{3}$ have a point in common, then it is possible to construct a shorter tour through $\left\{x_{1}, x_{2}, \ldots, x_{n}\right\}$. We can assume that $e_{1}$, with midpoint $m_{1}$, is oriented along the $x$ axis, the midpoint $m_{2}$ of $e_{2}$ lies above $e_{1}$, and the midpoint $m_{3}$ of $e_{3}$ lies above $e_{2}$. We can visualize the $e_{i}$ as illustrated in Figure 2, and note that there are two distinct cases that need to be considered.

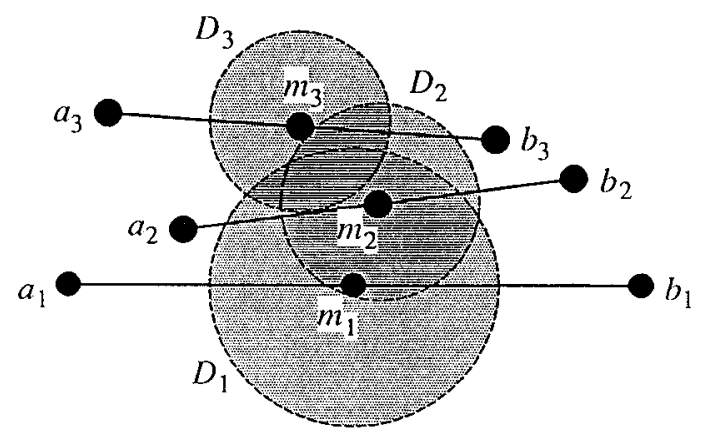

Figure 2. Three non-intersecting lines of a TSP tour and their $D_{i}$. Here, $\alpha=1 / 2$.

Since the endpoints of the $e_{i},\left\{a_{1}, a_{2}, a_{3}, b_{1}, b_{2}, b_{3}\right\}$, are distinct and are on the tour, there is a pair $a_{i}, b_{j}$ with $i \neq j$ such that $a_{i}$ and $b_{j}$ are joined by a path that contains none of the edges $e_{1}, e_{2}$, and $e_{3}$. The two cases depend on whether $|i-j|=1$ or $|i-j|=2$, c.f., the more schematic Figures $\mathbf{3 a}$ and $\mathbf{3 b}$.

In the case of $|i-j|=1$, we may assume that $i=3$ and $j=2$, as shown in Figure 3a. We form a new path by deleting $e_{2}$ and $e_{3}$ and adding the edges $\left(a_{2}, a_{3}\right)$ and $\left(b_{2}, b_{3}\right)$. By Lemma 1 , we can estimate the net change in the path length as

$$
\begin{aligned}
\Delta_{1} & =\left|a_{2}-a_{3}\right|+\left|b_{2}-b_{3}\right|-\left|e_{2}\right|-\left|e_{3}\right| \\
& \leq 2\left[\frac{1}{2}(\beta-1) r+6 \alpha \beta r\right]-2 r \\
& =(\beta-3+12 \alpha \beta) r
\end{aligned}
$$

since $\left|m_{2}-m_{3}\right|=\lambda \leq 2 \alpha \beta r$, for $D_{2} \cap D_{3} \neq \emptyset$. 
For the case of $|i-j|=2, i=1$ and $j=3$, as shown in Figure $3 \mathrm{~b}$. We now get a shorter tour by deleting all the $e_{i}$ and adding the edges $\left(b_{2}, b_{3}\right),\left(a_{3}, b_{1}\right)$, and $\left(a_{1}, a_{2}\right)$. The net change in weight is

$$
\begin{aligned}
\Delta_{2} & =\left|b_{3}-b_{2}\right|+\left|b_{1}-a_{3}\right|+\left|a_{2}-a_{1}\right|-\left|e_{1}\right|-\left|e_{2}\right|-\left|e_{3}\right| \\
& \leq 2\left[\frac{1}{2}(\beta-1) r+6 \alpha \beta r\right]+\beta r+2 \alpha \beta r-3 r \\
& =(2 \beta+14 \alpha \beta-4) r,
\end{aligned}
$$

where $\left|b_{3}-b_{2}\right|$ and $\left|a_{2}-a_{1}\right|$ are estimated as before and we estimate $\left|b_{1}-a_{3}\right|$ using the diagonal bound of Lemma 1 along with the fact that $\left|m_{1}-m_{3}\right|=\lambda \leq$ $2 \alpha \beta r$, since $D_{1} \cap D_{3} \neq \emptyset$.

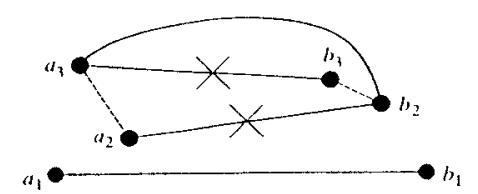

Figurc $3 \mathrm{~d}$ Rebunlding the $a_{2}$ to $b_{3}$ path when

$|\ell-\jmath|=1$ in Lemma 2 The cuived arc is a $p$ atth

the $X$ 'ed edges have been removed, and the

dashed edges have been added

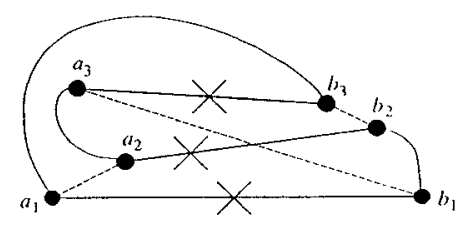

Figure 3b Rebuilding the tour when $|,-j|=2$

in Lenna 2 The cuived arcs are paths, he $X$ ' $\mathrm{ed}$

cdges have been removed, and the dashed edges

The choices $\beta=3 / 2$ and $\alpha=1 / 22$ are good enough to guarantee that $\Delta_{1}<0$ and $\Delta_{2}<0$.

\section{A Priori Edge-Length Bounds}

It is now easy to prove our main results. We label the edges of an optimal tour $T$ of $\left\{x_{1}, x_{2}, \ldots, x_{n}\right\} \subset[0,1]^{2}$ in order as $e_{1}, e_{2}, \ldots, e_{n}$, and we assume without loss that $n$ is even. We first construct disks $D_{i}$ of radius $\alpha\left|e_{i}\right|$ and center at the midpoint of $e_{i}$ for each $1 \leq i \leq n$, where $\alpha=1 / 22$. Let $\psi_{i}(\cdot)$ denote the indicator function of $D_{i}$, i.e., for all $x \in \mathbb{R}^{2}, \psi_{i}(x)=1$ if $x \in D_{i}$; otherwise $\psi_{i}(x)=0$. Let $A$ be the set of all odd $i$ such that $r \leq\left|e_{i}\right| \leq \beta r$, and let $B$ be the corresponding set of even $i$. We claim the following:

$$
\sum_{i \in A} \psi_{i}(x)+\sum_{i \in B} \psi_{i}(x) \leq 4 \psi(x)
$$

where $\psi(\cdot)$ is the indicator function of the square $[-1,2]^{2}$.

To prove the claim, we note that since we have assumed $n$ is even, pairs of edges with index belonging to $A$ do not share an endpoint; the same is true for pairs in $B$. For $\beta=3 / 2$ and $\alpha=1 / 22$, Lemma 2 tells us that no three disks of $A$ intersect and that no three disks of $B$ intersect. Hence, the point $x \in \mathbb{R}^{2}$ can belong to at most two disks associated with $A$ and two disks associated with $B$. Furthermore, since any disk with center in $[0,1]^{2}$ and radius bounded by $\alpha \beta r$ is contained in $[-\alpha \beta r, 1+\alpha \beta r]^{2} \subset[-1,2]^{2}$, we need only concern ourselves with $x \in[-1,2]^{2}$. This proves the claim.

If we now integrate (3.1) over $x$, we obtain a basic bound on a subset of the squared edge lengths of an optimal TSP tour:

$$
\sum_{r \leq\left|e_{i}\right| \leq \beta r}\left|e_{i}\right|^{2} \leq c
$$

where $c=36 \alpha^{-2} \pi^{-1}$. Finally,

$$
\begin{aligned}
\sum_{i=1}^{n}\left|e_{i}\right|^{2} & \leq 1+\sum_{n^{-1 / 2} \leq\left|e_{i}\right| \leq \sqrt{2}}\left|e_{i}\right|^{2} \\
& \leq 1+\sum_{k=1}^{m} \sum_{\beta^{k-1} n^{-1 / 2} \leq\left|e_{i}\right| \leq \beta^{k} n^{-1 / 2}}\left|e_{i}\right|^{2},
\end{aligned}
$$

where $m$ is the least integer $k$ such that $\beta^{k} n^{-1 / 2} \geq \sqrt{2}$. It suffices to take $m=\left\lceil\log _{3 / 2}(\sqrt{2 n})\right\rceil$, so applying (3.2) to (3.3) yields the bound

$$
\sum_{i=1}^{n}\left|e_{i}\right|^{2} \leq c_{1} \log n,
$$

where $c_{1}$ is constant as required by Theorem 1 .

Returning now to (3.1) and again integrating, we see that since $\left|e_{i}\right| \geq r$ for all $i \in A$ and $i \in B$,

$$
(|A|+|B|) \pi \alpha^{2} r^{2} \leq 36 .
$$


where $\psi(\cdot)$ is the indicator function of the square $[-1,2]^{2}$.

To prove the claim, we note that since we have assumed $n$ is even, pairs of edges with index belonging to $A$ do not share an endpoint; the same is true for pairs in $B$. For $\beta=3 / 2$ and $\alpha=1 / 22$, Lemma 2 tells us that no three disks of $A$ intersect and that no three disks of $B$ intersect. Hence, the point $x \in \mathbb{R}^{2}$ can belong to at most two disks associated with $A$ and two disks associated with $B$. Furthermore, since any disk with center in $[0,1]^{2}$ and radius bounded by $\alpha \beta r$ is contained in $[-\alpha \beta r, 1+\alpha \beta r]^{2} \subset[-1,2]^{2}$, we need only concern ourselves with $x \in[-1,2]^{2}$. This proves the claim.

If we now integrate (3.1) over $x$, we obtain a basic bound on a subset of the squared edge lengths of an optimal TSP tour:

$$
\sum_{r \leq\left|e_{i}\right| \leq \beta r}\left|e_{i}\right|^{2} \leq c
$$

where $c=36 \alpha^{-2} \pi^{-1}$. Finally,

$$
\begin{aligned}
\sum_{i=1}^{n}\left|e_{i}\right|^{2} & \leq 1+\sum_{n^{-1 / 2} \leq\left|e_{*}\right| \leq \sqrt{2}}\left|e_{i}\right|^{2} \\
& \leq 1+\sum_{k=1}^{m} \sum_{\beta^{k-1} n^{-1 / 2} \leq\left|e_{i}\right| \leq \beta^{k} n^{-1 / 2}}\left|e_{i}\right|^{2}
\end{aligned}
$$

where $m$ is the least integer $k$ such that $\beta^{k} n^{-1 / 2} \geq \sqrt{2}$. It suffices to take $m=\left\lceil\log _{3 / 2}(\sqrt{2 n})\right\rceil$, so applying (3.2) to (3.3) yields the bound

$$
\sum_{i=1}^{n}\left|e_{i}\right|^{2} \leq c_{1} \log n
$$

where $c_{1}$ is constant as required by Theorem 1 .

Returning now to (3.1) and again integrating, we see that since $\left|e_{i}\right| \geq r$ for all $i \in A$ and $i \in B$,

$$
(|A|+|B|) \pi \alpha^{2} r^{2} \leq 36 .
$$

But, $|A|+|B|=\left|\left\{i: r \leq\left|e_{i}\right| \leq \beta r\right\}\right|$, so

$$
\left|\left\{i: r \leq\left|e_{i}\right| \leq \beta r\right\}\right| \leq c r^{-2} \text {. }
$$

If we now write $\nu(n, t)$ as

$$
\begin{aligned}
\nu(n, t) & =\left|\left\{i:\left|e_{i}\right| \geq t\right\}\right| \\
& \leq \sum_{k=0}^{m_{t}-1}\left|\left\{i: \beta^{k} t \leq\left|e_{i}\right| \leq \beta^{k+1} t\right\}\right|,
\end{aligned}
$$

where $m_{t}=\min _{j}\left\{\beta^{j} t \geq \sqrt{2}\right\}$, we can use (3.6) to bound $\nu(n, t)$ as

$$
\begin{aligned}
\nu(n, t) & \leq c \sum_{k=0}^{m_{t}-1}\left(\beta^{k} t\right)^{-2} \\
& \leq c t^{-2} \sum_{k=0}^{\infty} \beta^{-2 k} \\
& =\frac{c}{1-\beta^{-2}} t^{-2}
\end{aligned}
$$

which is Theorem 2, with $c_{2}=c \beta^{2} /\left(\beta^{2}-1\right)$.

Corollary 3 now results from (3.8) by first noting that $n-\nu(n, x)$ is the number of edges in $E$ of length $x$ or less, then writing

$$
\begin{aligned}
\sum_{i \in E}\left|e_{i}\right| & =\sum_{\substack{e_{i} \in E \\
\left|e_{i}\right| \geq t}}\left|e_{i}\right|+\sum_{\substack{e_{i} \in E \\
\left|e_{i}\right|<t}}\left|e_{i}\right| \\
& \leq \frac{c_{2}}{t^{2}}+\int_{t}^{\sqrt{2}} x d(n-\nu(n, x)) .
\end{aligned}
$$

Integrating the right most term of (3.9) by parts then applying (3.8), we obtain

$$
\begin{gathered}
\int_{t}^{\sqrt{2}} x d(n-\nu(n, x))=\sqrt{2}(n-\nu(n, \sqrt{2})) \\
+t(\nu(n, t)-n)-n(t-\sqrt{2})+\int_{t}^{\sqrt{2}} \nu(n, x) d x \\
\leq \int_{t}^{\infty} \nu(n, x) d x \\
\leq c_{2} / t
\end{gathered}
$$

Inserting (3.10) into (3.9) and setting $t=|E|^{-1 / 2}$ yields Corollary 3 , with $c_{3}=c_{2}$.

\section{Concluding Remarks}

This paper investigates features of an optimal TSP tour that can be explicated without any knowledge of the locations of the points, and, in some cases, even without 
knowledge of the number of points. It is surprising that relatively tight bounds can be obtained under these conditions. Two of our main results bring bounds to the TSP that have been known for some time for the minimum spanning tree, but have been elusive for the TSP due to its $N P$-completeness. Our results also create a new open problem: Can the logarithmic term in Theorem 1 be removed?

The preceding arguments can be generalized to $d>2$, but, because of uncertainty concerning the logarithmic factor of Theorem 1, it seems inappropriate to give more than a sketch of a generalization to higher dimensions. The key idea is that in Lemma 2, we showed that if three of the $D_{i}$ associated with disjoint edges had a point in common, then we could find three disjoint edges $e_{1}, e_{2}$, and $e_{3}$ that were close together and nearly parallel. The existence of these edges then permitted us to construct a shorter tour. We used the fact that the $e_{i}$ did not cross to help provide bounds on the cost of modified paths; of course, such arguments are not available when $d>2$.

Still, if we consider the possibility that a large number $N(d)$ of $d$-spheres $D_{i}=D\left(m_{i}, \alpha\left|e_{i}\right|\right) \subset \mathbb{R}^{d}$ intersect and that the surface of any sphere in $\mathbb{R}^{d}$ can be covered with a finite number $M(\epsilon)$ of spherical caps with polar angle $\epsilon$, we can again show that we either have a bound like (3.1), with 4 replaced by a constant depending on $d$ and $\epsilon$, or else we will have three edges that are sufficiently parallel to permit an argument like that used to prove Lemma 2. In summary, one can prove

Theorem 3. There exist positive constants $c_{d}$ and $c_{d}^{\prime}$ such that for any traveling salesman tour $T$ of $\left\{x_{1}, x_{2}, \ldots, x_{n}\right\} \subset[0,1]^{d}$ and for all $n \geq 2$,

$$
\sum_{e \in T}|e|^{d} \leq c_{d} \log n
$$

and

$$
\nu_{d}(t)=|\{e \in T:|e| \geq t\}| \leq c_{d}^{\prime} / t^{d} .
$$

Just as in dimension two, there is a serious possibility that the logarithmic factor in (4.1) can be removed, but such an improvement does not seem to be obtainable by the present method.

We note that the methods of this paper are by no means restricted to the TSP; it is likely that they can be used to yield a priori inequalities for other problems, as well.

\section{References}

Few, L. (1955). "The shortest path and the longest road through $n$ points in a region," Mathematika 2 , 141-144.

Karloff, H.J. (1989). "How long can a Euclidean traveling salesman tour be?" SIAM J. Disc. Math. 2, 91-99.

Neumann, D.J. (1982). A Problem Seminar, SpringerVerlag, New York, NY.

Steele, J.M. (1990). "Probabilistic and worst-case analyses of classical problems of combinatorial optimization in Euclidean space," Mathematics of Operations Research 15, 749-770.

Steele, J.M. and Snyder, T.L. (1989). "Worst-case growth rates of some problems from combinatorial optimization," SIAM J. Computing 18, 278-287.

Verblunsky, S. (1951). "On the shortest path through a number of points," Proc. Amer. Math. Soc. 2. 904-913. 